\section{Evaluation of the antibacterial activity of bergamot essential oils on different Listeria monocytogenes strains}

Stefania M. Marotta, Filippo Giarratana, Alessio Parco, Domenico Neri, Graziella Ziino, Alessandro Giuffrida, Antonio Panebianco

Department of Veterinary Sciences, University of Messina, Messina, Italy

\section{Abstract}

Essential oils are aromatic and volatile substances extracted from plants and characterized by antimicrobial activity. The aim of the present study was to evaluate the antibacterial activity (agar disc-diffusion method) of seven different bergamot essential oils (BEOs) on eight Listeria monocytogenes strains. Minimal inhibitory concentration (MIC) of most efficient BEOs was estimated. Extremely variable results for agar disc-diffusion method for $L$. monocytogenes strains were reported. One of the tested microorganisms resulted insensible to all the BEOs; 3 strains showed an inhibition from weak to null and the remaining 4 a variable susceptibility. Among the BEOs tested, one showed a strong activity against four pathogenic strains. Four BEOs revealed weak, moderate or null activity in all the 7 sensitive strains, while for two oils only a weak or no activity was reported. MIC values were $0.625 \mu \mathrm{L} / \mathrm{mL}$ for the most efficient BE0, 2.5 and $5 \mu \mathrm{L} / \mathrm{mL}$ for the other samples that showed moderate inhibition. Experiment results are significantly related to the strains tested $(\mathrm{P}<0.01)$, rather than the BEO employed ( $P>0.01)$. In conclusion, we can consider BEO as a natural technological hurdle for Listeria monocytogenes in combination with other preservation strategies. Finally, this study underlines the necessity to evaluate the antimicrobial activity of EOs on a significant strains number of the same bacteria.

\section{Introduction}

The interest in natural methods that can make food safer, avoiding the use of chemical preservatives or additives, has increased the study on these products (Giarratana et al., 2013, 2016; Klein et al., 2013; Moreira et al., 2005; Muscolino et al., 2016). In this regard, essential oils (EOs), aromatic oily liquids obtained from plant materials, are well known for their preservative properties. These substances and their components are used in food, pharmaceutical and cosmetic industries, for their antibacterial, antifungal, antiviral, nematocidal, anti-carcinogenic and antioxidant properties (Frassinetti $e t$ al., 2011; Giarratana et al., 2014, 2015a, 2015b; Rota et al., 2008; Silva-Angulo et al., 2015). Bergamot essential oil (BE0), extracted from the peel of Citrus bergamia Risso, is characterized by several of these properties (Navarra $e t$ al., 2016; Pernice et al., 2009; Sicari et al., 2015; Trombetta et al., 2010). C. bergamia Risso is a typical fruit of southern Italy and its production is limited to the Ionian Sea coastal areas of Reggio di Calabria province (Sicari et al., 2015). BEO antibacterial and antiseptic activity is related to the presence of well-recognized antimicrobial compounds (Fisher and Phillips, 2006; Navarra et al., 2016; Pernice et al., 2009). These substances can be distinguished in volatile (e.g. limonene, linalool and linalyl acetate) and non-volatile (e.g. bergamottin, citroptene and bergaptene) components (Salvo et al., 2016). The major active EOs components are phenols, terpenes, aldehydes and ketones, whose action is performed against the cytoplasmic membrane of target microorganism cells (Hyldgaard et al., 2012). The hydrophobicity is also an important characteristic, which enables EOs to accumulate in cell membranes causing an increase of permeability until cell death (Moreira et al., 2005; Silva-Angulo et al., 2015). For all these reasons E0s employ in food technology is a concrete prospective for undesirable microbial flora control. The aim of this study was to evaluate the in vitro antimicrobial activity of different BEOs against several Listeria monocytogenes strains.

\section{Materials and Methods}

\section{Essential oils collection}

For this study, seven different samples of Citrus bergamia Risso essential oil (BE0) were tested. Among these, five were collected from local producers from Reggio Calabria district (BEOa-e), while, the remaining two from commercial products (BEOf: Mystic moments, Fordingbridge, UK; BEOg: Erboristeria magentina, Poirino, Italy).

\section{Bacterial cultures}

Eight Listeria monocytogenes strains were tested: five from seafood samples (wild types) and three from American Type Culture Collection (ATCC) (Table 1). Working cultures were prepared by inoculating a loopful from the frozen stock $\left(-80^{\circ} \mathrm{C}\right)$, on tryptic soy broth (Biolife, Milan, Italy) $+0.6 \%$ yeast extract (YE) (Biolife) and then incubated at $37 \pm 0.5^{\circ} \mathrm{C}$ for $24 \mathrm{~h}$, in order to achieve an $\mathrm{OD}_{600}$ of 1.2 , corresponding to $10^{9}$ colony forming unit/mL (SmartSpec Plus; Bio-Rad, Milan, Italy).
Correspondence: Stefania Marotta, Department of Veterinary Sciences, University of Messina, Polo Universitario della Annunziata, 98168 Messina, Italy.

Tel. +39.090.3503768.

Email:smarotta@unime.it

Conflict of interest: the authors declare no potential conflict of interest.

Key words: Listeria monocytogenes; Bergamot; Essential oils; Antibacterial activity.

Received for publication: 19 July 2016.

Revision received: 22 September 2016.

Accepted for publication: 22 September 2016.

This work is licensed under a Creative Commons Attribution-NonCommercial 4.0 International License (CC BY-NC 4.0).

(C)Copyright S.M. Marotta et al., 2016 Licensee PAGEPress, Italy

Italian Journal of Food Safety 2016; 5:6176 doi:10.4081/ijfs.2016.6176

\section{Determination of antimicrobial activity}

BEOs antibacterial activity was evaluated by agar disc diffusion method. Broth cultures strains were spread with a sterile swab on the surface of TSA (Biolife) $+0.6 \%$ YE agar plates. Sterile 6 -mm paper disc impregnated with 15 $\mu \mathrm{L}$ of each tested BEO $a-g$ were placed on the surface of inoculated plates. Two disks impregnated one with $15 \mu \mathrm{L}$ of Streptomycin (50 $\mu \mathrm{g} / \mathrm{mL}$ ) (Biolife) and one with $15 \mu \mathrm{L}$ of sterile distilled water as positive and negative control were used respectively. Plates were incubated at $37 \pm 0.5^{\circ} \mathrm{C}$ for $24 \mathrm{~h}$. Microbial inhibition was visually evaluated as the diameter of the inhibition zones surrounding the disks, including them, and recorded in millimeters according to NCCLS (2015). The antimicrobial activity of BE0 was divided into three ranges according to Rota et al. (2008): weak activity (inhibition zone $\leq 12 \mathrm{~mm}$ ), moderate activity (12 mm<inhibition zone $<20 \mathrm{~mm}$ ) and strong activity (inhibition zone $\geq 20 \mathrm{~mm}$ ).

\section{Determination of minimal inhibito-} ry concentration with broth dilu-

\section{tion assay}

BEOs with an antimicrobial activity from moderate to strong were tested for minimal inhibitory concentration (MIC) according to a modified NCCLS/CLSI standard method (NCCLS/CLSI, 2015). Serial two-fold dilutions of each BEO were made in a concentration ranging from 5 to $0.31 \mu \mathrm{L} / \mathrm{mL}$ in $10 \mathrm{~mL}$ sterile test tubes containing trypticase soy broth with $0.6 \%$ yeast extract. At this solution $5 \%(\mathrm{v} / \mathrm{v})$ Tween-20 (Biolife) was incorporated into the 
broth medium to enhance oil solubility. The inoculums were prepared from overnight broth cultures of sensitive strains (logarithmic growth phase cells). A 400- $\mu \mathrm{L}$ suspension of tested microorganisms was added to each tube. For positive and negative control we used two broth tubes containing respectively 50 $\mu \mathrm{g} / \mathrm{mL}$ of Streptomycin and only microorganism inoculums. MIC was assumed as the concentration in the lowest serial dilution of the BE0s that resulted in the lack of visible microorganism growth in tubes after $24 \mathrm{~h}$ incubation.

\section{Gas chromatography}

Analysis of most efficient BEO was carried out by as gas chromatography with flame ionization detection according to ISO 7609:1985 (ISO, 1985).

\section{Statistical analysis}

Each experiment was carried out in triplicate on two separate occasions. Results are expressed as mean values \pm standard deviation. One-way ANOVA test was performed to determine the mean significant differences among different BEOs treatment and strains tested, significance was assumed as $\mathrm{P}<0.01$ (XLSTAT, Microsoft Excel; Addinsoft, New York, NY, USA).

\section{Results}

\section{Antimicrobial activity: Listeria monocytogenes's variability}

Results are showed in Table 2. L. monocytogenes strains expressed a various range of susceptibility to BEOs action (Figure 1). In particular, among the 5 wild type strains, $115 \mathrm{me}$ resulted the most sensitive, revealing weak inhibition zones for $2 \mathrm{BEO}$, moderate sensibility against 4 oils and a strong reaction only for BEOd. Similar results are reported for $168 \mathrm{me}$, except that for no reaction to BEOg. Strain 94me demonstrated weak inhibition zones for most of BEO, moderate for BEOc and a strong susceptibility for BEOd. Listeria $157 \mathrm{me}$ showed a weak reaction only for $\mathrm{BEO} a, \mathrm{BEO} d$ and $\mathrm{BEO} e$, no inhibition zones were observed for others oils. Finally, 163me showed a potential resistance to BEOs antibacterial activity with no inhibition zones for all the oils employed. All the ATCC strains resulted completely insensitive to $\mathrm{BEO} e$; anyway, among them, ATCC 19111 resulted, overall, the most sensitive, showing a strong inhibition for $\mathrm{BEO}$, moderate for $\mathrm{BEO} c$ and weak for the other samples. Finally, the remaining ATCC 13932 and ATCC 7644 strains demonstrated a weak reaction to all the BEOs tested.

\section{Antimicrobial activity: bergamot essential oils' variability}

Among the seven BE0s employed in our study (Figure 2) BEOd was the most efficient in restricting $L$. monocytogenes growth. It was the only BEO characterized by a strong activity, with an inhibition diameter of $20 \mathrm{~mm}$ in $50 \%$ of the strains and weak inhibition zones in the remaining sensitive strains. Follows BEOc, with a moderate action on $50 \%$ of bacteria and a weak (25\%) to null (25\%) inhibition in the other strains. BEO $a$ resulted characterized by a mildly and weakly effective respectively on $37.5 \%$ and $50 \%$ of the strains studied. BEO $b$ action was moderate and weak in both $37.5 \%$ of strains, while BEOe showed an activity from weak to moderate in $50 \%$ of $L$. monocytogenes strains and no reaction in the left $50 \%$ including 163me and all the ATCC strains. Finally, BEOf and BEOg resulted the less effective showing only a weak activity respectively in the 75 and $62.5 \%$ of the strains and no inhibition in the remaining others.

\section{Determination of minimal inhibito- ry concentration}

$\mathrm{BEO} a, \mathrm{BEO} b, \mathrm{BEO} c, \mathrm{BEO} d$ and $\mathrm{BEO} e$, on the bases of antimicrobial activity, were selected for MIC determination. MIC values confirmed the results obtained by the agar disk diffusion method, as well as, their variable levels of inhibition. BEOd had the lowest MIC (0.625 $\mu \mathrm{L} / \mathrm{mL})$, BEO $a$ and BEO $c$ a value of $1.25 \mu \mathrm{L} / \mathrm{mL}$, while $\mathrm{BEO} b$ and $\mathrm{BEO} e$ the highest MIC (5 $\mu \mathrm{L} / \mathrm{mL})$.

\section{Chemical composition}

Seven compounds were detected in the most efficient Citrus bergamia Risso essential oil tested (BEO $d$ ). The compounds obtained and their abundance are in accordance with those reported by Navarra et al. (2015). The major compounds were hydrocarbons monoterpenes like Limonene $(35.5 \%)$ and Linalyl acetate (33.1\%); followed by Linalolo $(9.9 \%), \gamma$ Terpinene (6.9\%), $\beta$-Pinene $(5.9 \%), \quad \beta$ Bisabolene (0.6\%) and Geranile (0.2\%).

Table 1. Listeria monocytogenes strains.

\begin{tabular}{lcc} 
ID strains & Serotype & \multicolumn{1}{c}{ Origin } \\
$94 \mathrm{me}$ & - & Wild type - smoked salmon fillets \\
$115 \mathrm{me}$ & - & Wild type - smoked salmon fillets \\
\hline $157 \mathrm{me}$ & - & Wild type - smoked salmon fillets \\
$163 \mathrm{me}$ & - & Wild type - fresh salmon fillets \\
\hline $168 \mathrm{me}$ & - & Wild type - fresh salmon fillets \\
ATCC 13932 & $4 \mathrm{~b}$ & Human \\
\hline ATCC 19111 & $1 / 2$ & Poultry \\
ATCC 7644 & $1 / 2 \mathrm{c}$ & Human \\
\hline
\end{tabular}

ATCC, American Type Culture Collection.

Table 2. Bergamot essential oils' antimicrobial activity against the tested microorganisms.

\begin{tabular}{|c|c|c|c|c|c|c|c|c|}
\hline BEOs & $94 \mathrm{me}$ & $115 \mathrm{me}$ & $157 \mathrm{me}$ & $\begin{array}{l}\text { Strains } \\
163 \mathrm{me}\end{array}$ & 168me & ATCC19111 & ATCC13932 & ATCC7644 \\
\hline$a$ & $11 \pm 1$ & $14 \pm 1$ & $10 \pm 1$ & 0 & $14 \pm 0$ & $12 \pm 1$ & $11 \pm 1$ & $10 \pm 1$ \\
\hline$b$ & $12 \pm 0$ & $14 \pm 1$ & 0 & 0 & $16 \pm 1$ & $11 \pm 0$ & $8 \pm 1$ & $7 \pm 0$ \\
\hline$c$ & $15 \pm 1$ & $14 \pm 0$ & 0 & 0 & $16 \pm 1$ & $15 \pm 1$ & $10 \pm 0$ & $11 \pm 1$ \\
\hline$d$ & $20 \pm 0$ & $20 \pm 1$ & $10 \pm 1$ & 0 & $20 \pm 0$ & $20 \pm 1$ & $11 \pm 1$ & $11 \pm 1$ \\
\hline$e$ & $12 \pm 1$ & $14 \pm 1$ & $8 \pm 1$ & 0 & $12 \pm 1$ & 0 & 0 & 0 \\
\hline$f$ & $12 \pm 1$ & $12 \pm 0$ & 0 & 0 & $12 \pm 0$ & $10 \pm 1$ & $8 \pm 1$ & $8 \pm 0$ \\
\hline$g$ & $8 \pm 0$ & $8 \pm 1$ & 0 & 0 & 0 & $11 \pm 1$ & $9 \pm 1$ & $8 \pm 1$ \\
\hline
\end{tabular}

\footnotetext{
BEOs, bergamot essential oils; ATCC, American Type Culture Collection. Results are expressed as mean \pm standard deviation from the experiments in triplicate. The diameter of the disks ( $\emptyset=6$ mm) was included.
} 


\section{Discussion}

Terpenes antibacterial activity is well recognized. They pass through the cell wall and permeabilize cytoplasmic membrane, by destroying the multi-layers structure of polysaccharides, fatty acids and phospholipids. In bacteria, these events are associated with loss of ions and reduction of membrane potential, which lead to the proton pump collapse and depletion of ATP pool and lysis (Burt, 2004; Di Pasqua et al., 2007; Oussalah et al., 2007; Sikkema et al., 1995). It was reported that Listeria monocytogenes strains exposed to EOs activity react with the thickening and rupture of cellular membrane, and the progressive lack of cytoplasm material (Rasooli et al., 2006). Despite that, ANOVA test reported that our extremely variable results are significantly connected $(\mathrm{P}<0.01)$ to the strains tested; thus, to different cellular answers, when occurring injuring factors. It is well known that sublethal stressors can induce $L$. monocytogenes adaptation and develop of specific resistance against several substances such as antibiotics, bacteriophages and disinfectants (Fister et al., 2016; Kovacevic et al., 2016; Su et al., 2016). L. monocytogenes population is characterized by different stress robustness parameters that may represent an advantage in unfavorable condition. This pathogen, indeed, exhibits a mutable response upon stress exposure, which can be partially attributed to the presence of stable stress resistant variants (Metselaar et al., 2016). Some authors, also, suppose a multi-factorial genomic regulation to explain this various resistant patterns (Kovacevic et al., 2016). Indeed, we found a wild type strain (163me) total insensitive to oils action, probably due to an acquired resistance against lytic BEOs substances, whose mechanism of action has to be further investigated. Furthermore, we did not found any significant relation $(\mathrm{P}>0.01)$ between our results and the different types of BEO tested. Thus, different susceptibilities are, probably, imputable to the strains tested, rather than to oils compounds. Our best MIC result $(0.625 \mu \mathrm{L} / \mathrm{mL}-0.625 \% \mathrm{v} / \mathrm{v})$ is higher than value reported by Cirmi et al. (2016) $(0.125 \% \mathrm{v} / \mathrm{v})$ on a single $L$. monocytogenes strain.

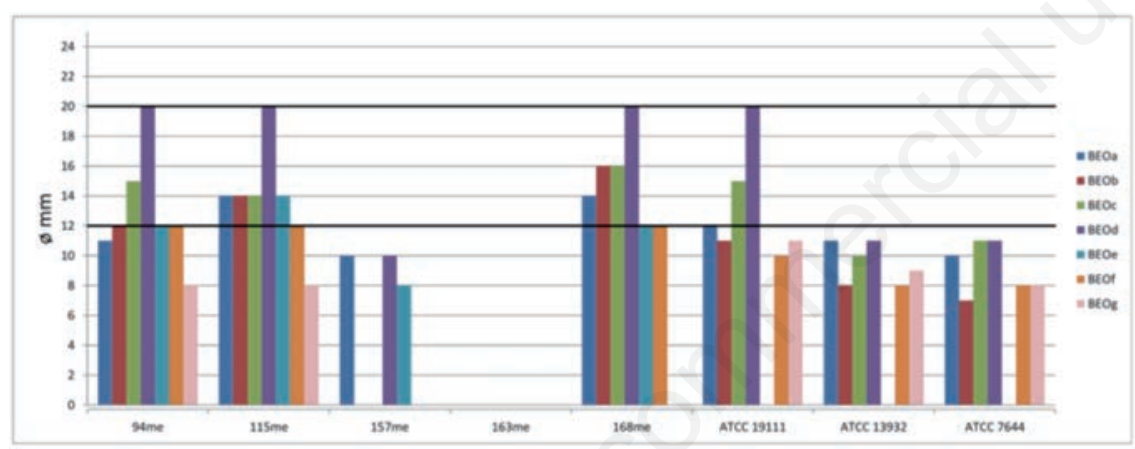

Figure 1. Listeria monocytogenes variable response to the activity of bergamot essential oils. Black lines represent critical limit fixed to assess oils' activity as weak, moderate or strong.

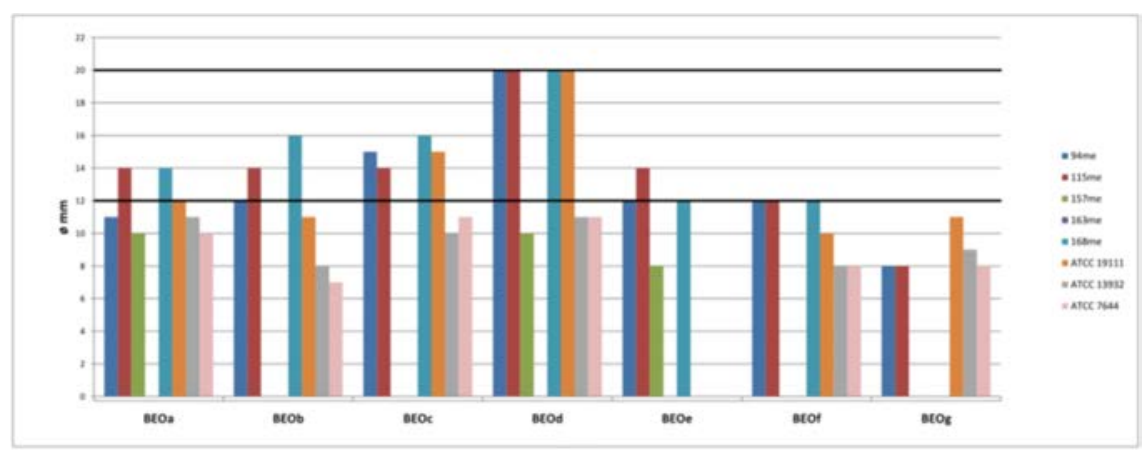

Figure 2. Bergamot essential oils' inhibitory activity on Listeria monocytogenes strains. Black lines represent critical limit fixed to assess oils' activity as weak, moderate or strong.

\section{Conclusions}

In conclusion, on the basis of our results, the different BEOs activities registered are mostly related to individual susceptibility of bacteria. Considering the extreme variability of Listeria monocytogenes's response to BEOs action, it is recommended to estimate their efficacy on a significative number of pathogenic strains in order to prospect a concrete employ in food industries as a valid natural alternative for the bio-control of the pathogen.

\section{References}

Burt S, 2004. Essential oils: their antibacterial properties and potential applications in foods: a review. Int J Food Microb 94:22353.

Cirmi S, Bisignano C, Mandalari G, Navarra M, 2016. Anti-infective potential of Citrus bergamia Risso et Poiteau (bergamot) derivatives: a systematic review. Phytother Res 30:1404-11.

Di Pasqua R, Betts G, Hoskins N, Edwards M, Ercolini D, Mauriello G, 2007. Membrane toxicity of antimicrobial compounds from essential oils. J Agr Food Chem 55:486370.

Fisher K, Phillips CA, 2006. The effect of lemon, orange and bergamot essential oils and their components on the survival of Campylobacter jejuni, Escherichia coli 0157, Listeria monocytogenes, Bacillus cereus and Staphylococcus aureus in vitro and in food systems. J Appl Microbiol 101:1232-40.

Fister S, Fuchs S, Stessl B, Schoder D, Wagner M, Rossmanith P, 2016. Screening and characterisation of bacteriophage P100 insensitive Listeria monocytogenes isolates in Austrian dairy plants. Food Control 59:108-17.

Frassinetti S, Caltavuturo L, Cini M, Della Croce CM, Maserti BE, 2011 Antibacterial and Antioxidant activity of essential oils from Citrus spp. J Essent Oil Res 23:27-31.

Giarratana F, Muscolino D, Beninati C, Giuffrida A, Panebianco A, 2014. Activity of Thymus vulgaris essential oil against Anisakis larvae. Exp Parasitol 142:7-10.

Giarratana F, Muscolino D, Beninati C, Ziino G, Giuffrida A, Panebianco A, 2013. Effects of thyme and rosemary essential oils on the microbiology and shelf life of Italian Mortadella. Fleischwirtschaft 93:183-7.

Giarratana F, Muscolino D, Panebianco F, Patania A, Benianti C, Ziino G, Giuffrida A, 2015a. Activity of R (+) limonene against Anisakis larvae. Ital J Food Safety 4:4.

Giarratana F, Muscolino D, Ragonese C, 
Beninati C, Sciarrone D, Ziino G, Mondello L, Giuffrida A, Panebianco A, 2016. Antimicrobial activity of combined thyme and rosemary essential oils against Listeria monocytogens in Italian mortadella packaged in modified atmosphere. $\mathrm{J}$ Essent Oil Res 2016:1-8.

Giarratana F, Panebianco F, Muscolino D, Beninati C, Ziino G, Giuffrida A, 2015b. Effect of allyl isothiocyanate against Anisakis larvae during the anchovy marinating process. J Food Protect 78:767-71.

Hyldgaard M, Mygind T, Meyer RL, 2012. Essential oils in food preservation: mode of action, synergies and interactions with food matrix components. Front Microbiol $3: 1-24$.

ISO, 1985. Essential oils. Analysis by gas chromatography on capillary columns. General method. ISO Norm 7609:1985. International Standardization Organization ed., Geneva, Switzerland.

Klein G, Rüben C, Upmann M, 2013. Antimicrobial activity of essential oil components against potential food spoilage microorganisms. Curr Microbiol 67:200-8.

Kovacevic J, Ziegler J, Wałecka-Zacharska E, Reimer A, Kitts DD, Gilmour MW, 2016. Tolerance of Listeria monocytogenes to quaternary ammonium sanitizers is mediated by a novel efflux pump encoded by emrE. Appl Environ Microb 82:939-53.

Metselaar KI, Abee T, Zwietering M H, den Besten HM, 2016. Modeling and validation of ecological behaviour of Listeria monocytogenes wild type and stress resistant variants. Appl Environ Microb 82:5389-401.

Moreira MR, Ponce AG, Del Valle CE, Roura SI,
2005. Inhibitory parameters of essential oils to reduce a foodborne pathogen. Food Sci Technol 38:565-70.

Muscolino D, Giarratana F, Beninati C, Ziino G, Giuffrida A, Panebianco A, 2016. Effects of allyl isothiocyanate on the shelf-life of gilthead sea bream (Sparus aurata) fillets. Czech J Food Sci 34:160-5.

Navarra M, Manucci C, Delbò M, Calapai G, 2016. Citrus bergamia essential oil: from basic research to clinical application. Front Pharmacol 6:36.

NCCLS, 2015. Methods for dilution antimicrobial susceptibility tests for bacteria that grow aerobically. Approved standard, document M07-A10. National Committee for Clinical Laboratory Standards, Wayne, PA, USA.

Oussalah M, Caillet S, Salmieri S, Saucier L, Lacroix M, 2007. Antimicrobial effects of alginate-based films containing essential oils on Listeria monocytogenes and Salmonella typhimurium present in bologna and ham. J Food Protect 70:901-8.

Pernice R, Borriello G, Ferracane R, Borrelli RC, Cennamo F, Ritieni A, 2009. Bergamot: a source of natural antioxidants for functionalized fruit juices. Food Chem 112:54550.

Rasooli I, Rezaei MB, Allameh A, 2006. Ultrastructural studies on antimicrobial efficacy of thyme essential oils on Listeria monocytogenes. Int J Infect Dis 10:236241.

Rota MC, Herrera A, Martinez RM, Sotomayor JA, Jordan MJ, 2008. Antimicrobial activity and chemical composition of Thymus vulgaris, Thymus zygis and Thymus hyemalis essential oils. Food Control 19:681-7.

Salvo A, Bruno M, La Torre GL, Vadalà R, Mottese AF, Saija E, Mangano V, Casale KE, Cicero N, Dugo G, 2016. Interdonato lemon from Nizza di Sicilia (Italy): chemical composition of hexane extract of lemon peel and histochemical investigation. Nat Prod Res 30:1517-25.

Sicari V, Loizzo MR, Branca V, Pellicanò TM, 2015. Bioactive and antioxidant activity from Citrus Bergamia Risso (Bergamot) juice collected in different areas of Reggio Calabria province, Italy. Int J Food Prop 1:10.

Sikkema J, De Bont JA, Poolman B, 1995. Mechanisms of membrane toxicity of hydrocarbons. Microbiol Rev 59:201-22.

Silva-Angulo AB, Zanini SF, Rosenthal A, Rodrigo D, Klein G, Martínez A, 2015. Comparative Study of the effects of citral on the growth and injury of Listeria innocua and Listeria monocytogenes cells. PloS One 10:e0114026.

Su X, Zhang J, Shi W, Yang X, Li Y, Pan H, Kuang D, Xu X, Shi X, Meng J, 2016. Molecular characterization and antimicrobial susceptibility of Listeria monocytogenes isolated from foods and humans. Food Control 70:96-102.

Trombetta D, Cimino F, Cristani M, Mandalari G , Saija A, Ginestra G, Speciale A, Chirafisi J, Bisignano G, Waldron $\mathrm{K}$, Narbad A, Faulds CB, 2010. In vitro protective effects of two extracts from bergamot peels on human endothelial cells exposed to tumor necrosis factor- $\alpha$ (TNF- $\alpha$ ). J Agr Food Chem 58:8430-6. 